\title{
TIC y mediaciones culturales en la educación superior: hacia un programa multidimensional
}

Juan Sebastián López López

Didier Arnulfo Santiago Franco

Recibido: 17 de julio de 2014 - Aprobado: 16 de octubre de 2014

\section{Resumen}

Tras una primera etapa de reflexión sobre la relación TIC-educación centrada en la brecha digital, se viene reconociendo la necesidad de estudiar, además, la cuestión de la capacitación necesaria para sacar provecho a los recursos tecnológicos disponibles. Sin embargo, se sigue prestando poca atención a la dimensión simbólica de la tecnología en general y de las TIC en particular, lo que genera serias dudas acerca del papel real que juega la cultura tanto en el discurso de la cultura digital como en el debate sobre la educación en la sociedad del conocimiento. En este sentido, el paso de la cultura como categoría residual a variable independiente surge de reconocer que: (a) las personas no sólo usan las tecnologías, sino que, sobre todo, las personalizan, las dotan de sentido y las hacen parte integral de su experiencia de mundo, y que (b) dichos procesos emergen no solo de experiencias subjetivas con las TIC, sino de robustas narrativas culturales en torno a las tecnologías. Este giro epistemológico hace posible un análisis multidimensional de las TIC en la educación, en el que la convergencia entre las dimensiones funcional y sociocultural de la tecnología arrojaría luces sobre la contradicción que muchos críticos de las nuevas tecnologías han puesto de manifiesto: la alta disponibilidad de recursos tecnológicos no garantiza mejores resultados en el plano cognitivo ni un enriquecimiento de nuestro capital cultural como sociedad. De ahí que la introducción de un programa cultural, ambicioso y dinámico, sea decisivo en el futuro de la investigación sobre educación superior y TIC.

Palabras clave: TIC, cultura, educación superior, símbolo, análisis multidimensional.

* $\quad$ Docente de la Universidad Santo Tomás, líder del proyecto de investigación "Habitar en espacios digitales" y candidato a Doctor en Investigación en Medios de Comunicación en la Universidad Carlos III de Madrid, España. Máster en Comunicación y Problemas Socioculturales de la Universidad Rey Juan Carlos de Madrid, España y Licenciado en Filosofía de la Universidad Santo Tomás de Colombia. Dirección Postal: Carrera 9, 51-11, Bogotá, Colombia. Correo electrónico: sebastianlopez@usantotomas.edu.co

** Docente de la Universidad Santo Tomás y la Corporación Universitaria Minuto de Dios. Orienta las cátedras de Tecnología y Educación, y un seminario sobre John Dewey. Investigador en Filosofía para Niños. Magister en Filosofía de la Universidad del Rosario. Licenciado en Filosofía de la Universidad Santo Tomás. Dirección Postal: Carrera 9 , 51-11, Bogotá, Colombia. Correo electrónico: didiersantiago@usantotomas.edu.co 


\title{
ICT and cultural mediation in higher education: towards a multidimensional program
}

\author{
Juan Sebastián López López* \\ Didier Arnulfo Santiago Franco*
}

\section{A bstract}

Following an initial reflection stage with regard to the ICT- education relationship centered on the digital gap, there is a recognized need to also study the question with regard to the necessary training to take advantage of the available technological resources. However, little attention is being paid to the symbolic dimensión of technology in general and ICT in particular, which generates seroius doubts regarding the real role played by culture in the discourse regarding digital culture as well as in the debate with regard to education in the society of knowledge. In this sense, the change of culture as a residual category to independent variable comes from recognizing that: (a) people do not only use technologies, but above all they personalize them, they endow them with sense and make them an integral part of their experience of the world. And that (b) said processes emerge not only from subjective experiences with ICT, but from robust cultural narratives having to do with technologies. This epistemological turn of events makes possible a multidimensional analysis of ICT in education, in which the convergence between the functional and sociocultural dimensions of technology would shed light on the contradiction that many critics of the new technologies have made manifest: the high availability of technological resources does not guarantee better results in the cognitive plane or an enrichment of our cultural capital as a society. From this it is clear that the introduction of an ambitious and dynamic cultural program needs to be decisive in the future of research having to do with ICT and higher education.

Keywords: ICT, culture, higher education, symbol, multidimensional analysis.

Professor at the Universidad Santo Tomás, leader of research project "Inhabiting digital spaces"; and PhD candidate in Research in Communication media at Universidad Carlos III de Madrid, Spain. Masters in Communication and Socio-Cultural problems of the Universidad Rey Juan Carlos de Madrid, Spain, and bachelor's in Philosophy from the Universidad Santo Tomás of Colombia. Address: Carrera 9, 51-11. Email: sebastianlopez@usantotomas.edu.co.

* Professor at the Universidad Santo Tomás and at the Corporación Universitaria Minuto de Dios. Orients the subjects of Technology and Education, and a seminar regarding John Dewey. Investigator in Philosophy for children. Masters in Philosophy from the Universidad del Rosario. Bachelors in Philosophy from the Universidad Santo Tomás. Address: Carrera 9, 51-11. Correo electrónico: didiersantiago@usantotomas.edu.co. 


\title{
TICS et médiations culturelles en enseignement supérieur: vers un programme multidimensionnel
}

\author{
Juan Sebastián López López* \\ Didier Arnulfo Santiago Franco*
}

\section{Résumé}

Après une première étude de réflexion sur la relation TICS- éducation fondée sur la fracture numérique, on reconnait de plus en plus la nécessité d'étudier, en outre, la question de la formation nécessaire pour tirer profit des ressources technologiques disponibles. Cependant, l'on continue d'accorder moins d'attention à la dimension symbolique de la technologie en général, à celle des TICS en particulier, ce qui soulève des doutes sérieux sur le rôle réel de la culture d'une part, lors du discours de la culture numérique et d'autre part lors du débat sur l'éducation dans la société de la connaissance. En ce sens, le passage de la culture de catégorie résiduelle à variable indépendante vient de la reconnaissance du fait que (a) les gens n'utilisent pas seulement les technologies, mais, surtout, ils les personnalisent, leur donnant et les intègrent à leur expérience du monde et que (b) ces processus émergent non seulement des expériences subjectives avec les TICS, mais des récits culturels solides autour de la technologie. Ce tour épistémologique rend possible une analyse multidimensionnelle des TICS dans l'éducation, où la convergence entre les dimensions fonctionnelles et socioculturelles de la technologie permettrait de faire la lumière sur la contradiction que de nombreux détracteurs des nouvelles technologies ont montré: la haute disponibilité des ressources technologiques ne garantit pas de meilleurs résultats sur le plan cognitif ni l'enrichissement de notre capital culturel en tant que société. C'est pourquoi, la mise en marche d'un programme culturel ambitieux et dynamique, est crucial pour l'avenir de la recherche sur l'enseignement supérieur et TICS.

Mots clés: TICS, culture, enseignement supérieur, symbole, analyse multidimensionnel.

\footnotetext{
Enseignant de l'Université Santo Tomás, leader du projet de recherche "Vivre dans des espaces numériques" et candidat au doctorat en recherche des médias auprès de l'Université Carlos III de Madrid, Espagne. Master en Communication et Problèmes Socioculturels de l'Université Rey Juan Carlos de Madrid, Espagne et Licencié en Philosophie de l'Université Santo Tomás de Colombie. Adresse: Carrera 9, 51-11. Email: sebastianlopez@usantotomas.edu.co.

* Enseignant de l'Université Santo Tomás et de la "Corporación Universitaria Minuto de Dios". Dispense les chaires Technologie et d'Éducation, et un séminaire sur John Dewey. Chercheur en Philosophie pour enfants. Master en Philosophie de l'Université del Rosario. Licencié en Philosophie de l'Université Santo Tomás. Adresse: Carrera 9, 51-11. Email: didiersantiago@usantotomas.edu.co.
} 


\section{Introducción}

$\mathrm{Al}$ interior de la comunidad académica se viene estudiando con cada vez más fuerza el nuevo paisaje mediático que Internet ha ayudado a consolidar (Jenkins, 2008:2009; Ardévol et al. 2010; Ito et al. 2009; Scolari, 2010). Ese interés creciente por el fenómeno digital puede deberse a la constatación de que las nuevas tecnologías de la información y la comunicación han posibilitado una serie de cambios profundos en todos los niveles de la sociedad (Castells, 2007; 2010). En ese orden de ideas, no sorprende que haya cada vez más investigaciones desde el campo educativo sobre cambio social y tecnológico, brecha digital, alfabetización mediática o competencias digitales (Davies et al. 2011; Ferres y Piscitelli, 2011; Cobo, 2009).

Junto a estas líneas de análisis, también es posible advertir, de un tiempo para acá, una perspectiva cada vez más robusta que pretende integrar la cultura a la ecuación TIC-educación (Balsamo, 2011; Kangas, 2011; Ardévol et al. 2010; Ito et al. 2009; Jenkins, 2008; 2009; 2009b). Por esta vía, se han obtenido importantes resultados que evidencian cómo ciertas dinámicas culturales reconfiguran la vida social. Por lo tanto, esa atención prestada a la cultura exige plantearse la posibilidad de hacer converger todos estos resultados empíricos con una teoría multidimensional de las TIC y la educación. Y es que, debido a su fuerte carga cognitivista y racionalista, los vínculos entre estructura cultural y acción comunicativa que viene elaborando la comunicología carecen en muchos casos de fuerza explicativa, por lo que esa relación TIC-educación divaga con frecuencia entre el voluntarismo y el determinismo tecnológico, y entre la nostalgia y el optimismo cultural.

En este orden de ideas consideramos pertinente proponer un programa "fuerte" de análisis cultural para el estudio de las prácticas mediáticas en contextos de educación superior. Dicho programa estaría sustentado en dos principios que estructuran lo que J. Alexander denomina "sociología cultural". El primero de ellos es que los objetos de estudio de la ciencia social participan como significantes en sistemas de significados dobles o binarios. El segundo, que la acción social puede ser entendida como un performance; es decir, que es esencialmente discursiva y está cargada de pretensiones simbólicas (Reed y Alexander, 2009, p. 30).

Así pues, para llevar a cabo un estudio culturalmente reconstruido del papel de las TIC en la educación superior, será necesario determinar, en primer lugar, cuáles son los significados atribuidos tanto a las TIC como a las prácticas mediadas por estas en el caso de estudiantes universitarios. Consideramos que, por esta vía, es posible arrojar luces sobre un problema teóricamente conflictivo para las ciencias sociales y los estudios en educación: la relación entre las estructuras culturales (en este caso relativas al aprendizaje y las TIC) y las interpretaciones que hacen los actores sociales (en este caso estudiantes universitarios) de esa estructura, y que dirigen y orientan su acción (Alexander, 2008, p. 160). 


\section{Planteamiento del problema}

Tras una primera etapa de reflexión sobre la relación TIC-educación centrada en la brecha digital, se viene reconociendo la necesidad de estudiar, además, cuáles serían las competencias necesarias para sacar provecho a los recursos tecnológicos disponibles. Sin embargo, se sigue prestando poca atención a la dimensión simbólica de la tecnología en general y de las TIC en particular, lo que genera serias dudas acerca del papel real que juega la cultura tanto en el discurso de la cultura digital como en el debate sobre la educación en la sociedad del conocimiento (Unesco, 2005).

En este sentido, el necesario paso de la cultura como categoría residual a variable independiente que aquí sugerimos como programa para un estudio más integral de la relación TIC-educación, parte de reconocer, primero, que las personas no solo usan las tecnologías, sino que, sobre todo, las personalizan, las dotan de sentido y las hacen parte integral de su experiencia de mundo (Baudrillard, 2012) (Melucci, 2001) y, segundo, que dichos procesos emergen no solo de experiencias subjetivas con las TIC, sino de robustas narrativas culturales que anteceden e informan nuestra relación con la tecnología (Alexander, 2003). De ahí que desde un análisis multidimensional de las TIC en la educación se ensanchen las posibilidades de hacer frente a la evidencia de que la alta disponibilidad de recursos tecnológicos no garantiza mejores resultados en el plano cognitivo (Barrera-Osorio y Linden, 2009) ni un necesario crecimiento de nuestro capital cultural (Wolton, 2000).

En este orden de ideas, nuestra propuesta se estructura en torno a tres hipótesis:

1. Existe una contradicción entre los niveles de inmersión en entornos tecnológicos y mediáticos de los estudiantes en educación superior y el poco uso que estos le dan a las TIC para fines académicos e investigativos. Puede que ello se deba a que los estudiantes asocian las TIC a valores negativos (dispersión, distracción, aislamiento, etc.) y que en ese proceso influyan las posturas de los docentes y de la misma Universidad, a su vez informadas por un debate helicoidal a nivel de teoría social entre apocalípticos e integrados tecnológicos.

2. Por tanto, para dar cuenta de por qué no hay aún una correlación directa entre acceso a TIC y usos innovadores en términos académicos e investigativos, es necesario estudiar los significados, valores y creencias atribuidos a las TIC. Solo así se podrá comprender mejor cuál es el entorno simbólico que configura las prácticas mediáticas de los estudiantes universitarios y que da forma a su relación con las tecnologías.

3. Integrar el nivel de los significados atribuidos a los niveles de acceso y usos académicos e investigativos de las TIC daría lugar, entonces, a concretar una lectura multidimensional del debate sobre la brecha digital en la educación superior. Ello permitiría a las instituciones educativas interesadas en esta 
perspectiva reconstruir sus planes de alfabetización y sus políticas sobre TIC, en pro de un mejoramiento profundo e integral de la competencia digital de su comunidad académica.

Con esto en mente, a continuación describiremos algunas bases conceptuales que dan forma este programa analítico aplicado en el contexto específico de la comunidad estudiantil de la Universidad Santo Tomás de Aquino de Bogotá.

\section{Perspectiva teórica}

\section{El hombre capaz: decir, actuar y narrar}

La primera coordenada teórica que vale la pena describir es la noción de hombre capaz de Paul Ricoeur. Según esta noción, la persona es su historia, una historia marcada por la capacidad de decir, actuar y contar (Ricoeur, 2005): poder decir, dada la capacidad de las personas de producir un discurso sensato; poder actuar, como la capacidad de producir acontecimientos en la sociedad y la naturaleza; y poder contar, como el arte milenario de contar historias. En palabras de Ricoeur: "los acontecimientos de cualquier origen solo se vuelven legibles e inteligibles cuando se cuentan dentro de una historia" (Ricoeur, 2005).

Por ende, para comprender la compleja relación que generan las personas con la tecnología desde la noción de hombre capaz, habrá que indagar en los discursos producidos, las acciones realizadas y las historias relatadas. En el caso concreto de los estudiantes, ello permite acceder a un nivel rico en datos y muy poco explorado: las experiencias que estos van elaborando con sus pares y su relación con los sistemas tecnológicos y socioculturales que funcionan como entorno de esas experiencias (Ricoeur, 2000, p. 194). En términos hermenéuticos, se trataría de analizar si los motivos de los agentes ${ }^{1}$ y las reglas de configuración de la realidad poseen un sustrato simbólico, y si dicho sustrato, por consiguiente, resulta ser la clave interpretativa de su acción en lo que respecta a las TIC.

Ese sustrato simbólico y cultural es desarrollado por Ricoeur a través de su noción de identidad narrativa ${ }^{2}$ que surge desde las relaciones que se generan entre la vida y la narración. Según Ricoeur, la vida es el tiempo que transcurre entre el nacimiento y la muerte, de ahí que la vida pueda ser pensada como un texto en el que las personas unifican el relato histórico con el relato de ficción.

1 El término agencia implica la capacidad que tiene una persona de efectuar cambios en el mundo físico y social al mismo tiempo que se reconoce como productor de dichas acciones. En el ámbito de la tecnología es revisar el cómo los agentes tienen la capacidad de producir tecnologías, apropiarse de ellas y construir nuevas formas de narración que configuran la realidad social.

2 La noción de identidad narrativa es elaborada por Paul Ricoeur en la última parte de Tiempo y narración (Ricoeur, 2006, pp. 994-1002), en una conferencia que pronuncia con motivo de su doctorado Honoris Causa en la Universidad de Neuchâtel (Ricoeur, 1986), en Sí mismo como otro (Ricoeur, 1996), como también en el texto La vida: un relato en busca de un narrador (2006). 
Ricoeur busca restablecer la separación que ha habido entre el relato y la vida, pues "el saber parece alejar el relato de la vida en tanto que vivida y que confina al relato en el campo de la ficción" (Ricoeur, 2006, p. 9).

El tipo de sujeto del que parte Ricoeur para enunciar su noción de identidad narrativa es un individuo histórico, finito y reflexivo que se designa como agente, locutor y enunciador (Ricoeur, 1986). En ese sentido, el análisis del ser humano parte de la reflexividad e interpretación de sí mismo, de su narración, de los otros y de las instituciones. Así, el hombre pasa por tres momentos: primero, al reflexionar sobre sí mismo se reconoce como autor de sus propias acciones, al mismo tiempo que evidencia la imbricación de su historia personal con la historia de otros (padres, amigos, compañeros trabajo etc.); segundo, en el encuentro con los otros los reconoce como agentes capaces, los ve a la cara y les dice: ¡tú también eres capaz!, jtú eres como yo!; tercero, las instituciones se convierten para el sujeto en garantes de la vida en comunidad, pues representan una estructura de convivencia que no puede ser reducida a las relaciones interpersonales (Moratalla, 2007, p. 291).

Podría afirmarse, entonces, que desde estos tres escenarios de relación en los que el hombre se desarrolla, la tecnología deviene como un gran símbolo a la luz del cual el hombre puede interpretar sus relaciones personales, sociales e institucionales. No puede perderse de vista que, para Ricoeur, el simbolismo confiere a la acción una primera legibilidad, y hace de la acción un cuasi-texto para el cual los símbolos proporcionan las reglas de significación en función de las cuales tal comportamiento se puede interpretar (Ricoeur, 2006, p. 18). En otras palabras, los símbolos pueden operar como signos de reconocimiento entre miembros de una comunidad ${ }^{3}$. De este modo, la tecnología puede convertirse en una forma de interpretación y comprensión de la realidad personal, social e institucional.

\section{Sociología cultural y performance social}

Siguiendo la línea de los planteamientos de Ricoeur, la sociología cultural (SC) que lidera Jeffrey Alexander insistirá en la necesidad de vincular los niveles macro de la estructura y el orden social con los niveles micro de las interacciones y la acción individual, así como los medios teóricos "no fácticos" con los medios empíricos "fácticos" del continuum del pensamiento científico (Alexander, 2000b). Por esta vía, la SC buscará superar polaridades recurrentes en el pensamiento sociológico, como las de acción instrumental versus acción cultural, y orden cooperativo versus orden coercitivo. Al respecto, afirma Alexander (2005, p. 2):

We need to do away with the traditional concepts of action and structure, and to dissolve, not reify, such dichotomies as culture versus institution and instrumental versus normative (Alexander, 2005).

3 Sobre esta afirmación del símbolo como forma de reconocimiento. Cfr. Ricoeur, Le destinataire de la religion: I'homme capable (2013). 
Desde su perspectiva, entonces, no es posible considerar los hechos sociales de un modo naturalista, como cosas que pueden existir en y por sí mismas, sin la mediación de códigos culturales (Alexander, 2000, p. 169). De hecho, afirma que el análisis de los significados debe complementar el de las dimensiones materiales del objeto de estudio. Para Alexander no será necesario un retorno al materialismo para explicar la materia $(2009$, p. 8), por el contrario, afirmará que el poder explicativo de esta versión reconstruida de las ciencias sociales radica en asumir la cultura como constitutiva de lo social (Reed y Alexander, 2009).

Es en ese nivel de la investigación social donde las humanidades vienen a ocupar, a juicio de Alexander, un lugar central tanto en términos de sus objetos de estudio como de las herramientas teóricas e investigativas que han desarrollado para atender al valor del significado en la vida social. Alexander afirmará que a partir del estudio de la estética y de la actuación teatral se podrán entender las formas como se construyen modos de experiencia entre los individuos y los objetos que les rodean, así como los alcances simbólicos de su acción. De ahí que Alexander $(2009$, p. 7$)$ se pregunte, con respecto a la tecnología avanzada, si podemos medir su impacto solo en función de su potencia en el procesamiento de datos y de su rol económico y político:

...en tanto entidad con respecto al sistema social, puede tocarse, medirse, racionalizarse; pero con relación al sistema cultural del que hace parte, es un significado y un significante en el que los actores no pueden separar su estado subjetivo de su mente (...). La tecnología debe aprender a verse como un discurso, como un sistema de signos que está sujeto a imperativos semióticos y abierto a demandas sociales y psicológicas (Alexander, 2000, p. 185).

Podría afirmarse, entonces, que para Alexander las tecnologías contienen una carga estética (textual e icónica) que impacta profundamente la vida social y cultural contemporánea y que es fundamental para recuperar el sentido de su dimensión material (2008b). De ahí que insista, en primer lugar, en atender a las experiencias icónicas y estéticas en las que nos introducen las tecnologías:

Iconographic experience explains how we feel part of our social and physical surroundings, how we experience the reality of the ties that bind us to people we know and people we don't know, and how we develop a sense of place, gender, sexuality, class, nationality, our vocation, indeed our very selves (Alexander, 2008c, p. 7).

Por otra parte, afirma con respecto no solo al carácter estético de las tecnologías en sí mismas sino a los alcances simbólicos de las prácticas tecnológicas, que tampoco se puede perder de vista la relación analítica que estas guardan con las estructuras culturales de la sociedad en general, en los modos como se ordenan en torno a las nociones de éxito o fracaso. Alexander otorga a este marco de evaluación (éxito o fracaso) una importancia enorme a la hora de comprender cuál es la finalidad que persigue toda acción en un mundo caracterizado por interacciones sociales tecnológicamente mediadas. Con ello se persigue, en sus 
propias palabras, "establecer qué hace al significado importante, para entender qué hace a algunos hechos sociales plenamente significativos" (Alexander, 2005b, p. 9). Por ello, afirmará que la comunicación entre actores, y por consiguiente las posibilidades de éxito o fracaso en el posicionamiento de un significado, estriban en re-fusionar la realidad por medio de la encarnación de guiones que puedan llegar a ser socialmente compartidos:

Communication of belief is accomplished, metaphorically speaking, by becoming an actor in a script. Then, you need to get the others who constitute the putative audience for your action to take this script as real, to experience it, not as a "script," not as symbolic or contrived, but as completely real, as having an ontological status (...). They must accept your symbolic projection. They must speak your language, so that you are both reading from the same page (Alexander, 2005, p. 3).

En ese sentido, su perspectiva se aleja de postulados teóricos que entienden la acción social como desde un punto de vista racional y del interés particular. Por el contrario, para la SC la acción siempre será significativa y estará inscrita en estructuras culturales socialmente compartidas que informan, incluso, las nociones de pragmatismo que elaboran los actores sociales. De ahí que Alexander afirme con respecto a la investigación social:

Researching society empirically involves, instead, a complex double reading. Social actors are 'reading' reality, moving pragmatically in relation to their meaning systems, and we are 'reading them', trying to get inside their own arrangements by using our own meanings. When we do so successfully, we have the beginnings of a sociological explanation (Alexander, 2009, p. 34).

En este orden de ideas, Alexander (2005; 2005b) propone estudiar la acción social desde un paradigma que él denomina "performance social". Grosso modo, estas serían las principales dimensiones de análisis de una acción social entendida en términos performativos:

1. Los sistemas de representaciones colectivas, entendidos como "mundos (que) proveen los símbolos de fondo para las actuaciones sociales" (Alexander, 2005b; p. 21).

2. Los actores que como "intérpretes o actuantes tienen subjetividades internas que manifiestan sus particularidades e identidades sociales y que reflejan su estatus socialmente definido" (Ídem).

3. Las audiencias, en tanto "observadores de la actuación cultural. Ellos decodifican lo que los actores han codificado pero lo hacen de formas variadas" (Ídem).

4. Los medios de producción simbólica, entendidos como "las cosas materiales mundanas sobre las cuales se pueden hacer proyecciones. Ellos (los actores) 
necesitan objetos que sirvan como representaciones iconográficas, para que los ayuden a dramatizar y hacer vívidos los símbolos invisibles que están tratando de representar" (Ídem, p. 22).

5. Puesta en escena. Se asume como el proceso en el que los actos son "codificados, narrados, y retóricamente configurados (...). La actuación tiene una secuencia temporal, está coreografiada espacialmente y obedece leyes artísticas que reflejan estas exigencias" (Ídem).

6. Poder social, que "afecta profundamente el proceso de performance (...). No todos los textos son igualmente legitimados (...). No a todas las actuaciones, y no a todas las partes de una actuación en particular se les permite proceder" (Ídem).

\section{Diseño metodológico}

Desde el marco teórico esbozado líneas arriba, y a fin de ir concretando algunos enunciados teóricos en estrategias metodológicas, diseñamos un modelo de trabajo de campo que nos permitiera estudiar las representaciones simbólicas en torno a las TIC y las prácticas mediáticas de algunos grupos de estudiantes de la Universidad Santo Tomás. Escogimos trabajar con estudiantes debido a que consideramos que son ellos el centro del proceso académico (al menos en lo que toca a la educación superior). Nuestro primer objetivo fue el buscar relaciones entre una supuesta estructura cultural y las prácticas comunicativas de los estudiantes a través de una serie de relatos asociados a las TIC y el mundo actual. De esa forma intentaríamos establecer vínculos entre construcciones simbólicas colectivas y usos personales y comunitarios de las TIC.

También nos interesaba comprender si las prácticas mediáticas de los estudiantes con los que trabajamos estaban impregnadas de significado para, de ser así, tratar de establecer sus contenidos. Por tanto, quisimos indagar en los siguientes niveles:

1. Guiones. El entramado compuesto por cultura, códigos, narrativas básicas y configuraciones retóricas que enmarcaban las prácticas mediáticas de los estudiantes y que sirven de fondo a sus prácticas mediáticas.

2. Las interpretaciones mismas de los estudiantes sobre estos guiones y narrativas.

3. Las tecnologías y medios que los estudiantes privilegian y con los que se relacionan de manera profunda e intensa, construyendo patrones de uso, representaciones significativas y narraciones.

4. Las intenciones, intereses e influencias personales o grupales que ordenan las prácticas mediáticas de los estudiantes. 
5. Los procesos de negociación y generación de discursos en torno a los usos apropiados, legítimos y productivos de las TIC entre docentes y estudiantes.

6. Las relaciones entre pares (estudiante-estudiante).

7. El acceso a tecnologías.

8. Los procesos de re-fusión más exitosos por parte de estudiantes en lo que atañe a sus prácticas mediáticas de tipo lúdico y académico.

Así pues, seleccionamos ocho grupos constituidos por estudiantes de distintos programas de la USTA, que cursaban en ese entonces las cátedras de Filosofía Institucional ( $1^{\circ}$ semestre), Epistemología $\left(6^{\circ}\right.$ semestre) y Ética ( $9^{\circ}$ semestre), todas ellas pertenecientes al núcleo de formación integral del Departamento de Humanidades. La elección de estos grupos obedeció a que, en nuestro criterio, el carácter transversal de las cátedras de humanidades garantizaba en la práctica un alto grado de diversidad en la procedencia disciplinar de los estudiantes que las conforman, así como un alto grado de pluralidad en sus opiniones.

A fin de cumplir con las metas que nos habíamos trazado, nos decantamos por llevar a cabo ocho sesiones de grupos focales sobre dos grandes niveles de indagación que denominamos: (1) tecnología, sociedad e individuo, y (2) negociación entre actores. Establecimos también una serie de tópicos de indagación que respondían a las inquietudes que a nivel teórico nos habíamos planteado hasta entonces. Por esta vía pretendíamos estudiar las representaciones simbólicas relativas a las TIC que pudieran ser influyentes en los estudiantes de la USTA y, siguiendo a Ito et al. (2009), indagar en los cambios profundos que acontecen en entornos digitales, específicamente en las formas como los estudiantes negocian lo relativo al ocio, el aprendizaje y la producción de conocimiento (ver tabla 1).

Una vez empezamos a obtener los datos fruto de las sesiones grupales, procedimos a revisar la información y a codificarla. A continuación expondremos algunos de los resultados que nos parecieron más interesantes, acompañados de algunas intervenciones puntuales de los estudiantes. Con esto pretendemos estimular las posibles interpretaciones de los lectores de este texto, a la vez que evitar, al menos en principio, la formulación de categorías muy cerradas. Valga decir que los datos aportados por los estudiantes han sido tan densos e interesantes que aún estamos en fase de reflexión sobre los mismos. Por eso, insistimos, se intentará respetar al máximo las intervenciones de ellos.

\section{Resultados}

En principio, parecería que los estudiantes tienen una relación naturalizada con las TIC. Esto quiere decir que, al parecer, sus prácticas comunicativas, relaciones sociales y tareas cotidianas se llevan a cabo a través de mediaciones tecnológicas sin que la tecnología en sí misma llegue a ser objeto de reflexión significativa. 
Tabla 1. Plan de trabajo con grupos focales

\begin{tabular}{|c|c|c|}
\hline \multirow[b]{2}{*}{ TÓPICO DE INDAGACIÓN } & \multicolumn{2}{|c|}{ FOCUS GROUPS CON ESTUDIANTES } \\
\hline & $\begin{array}{l}\text { No. 1. TECNOLOGÍA, } \\
\text { SOCIEDAD E INDIVÍDUO }\end{array}$ & $\begin{array}{l}\text { No. 2. NEGOCIACIÓN } \\
\text { ENTRE ACTORES }\end{array}$ \\
\hline $\begin{array}{l}\text { Representaciones generales en } \\
\text { torno a la tecnología }\end{array}$ & \multicolumn{2}{|c|}{$\begin{array}{l}\text { Intenta definir la palabra "tecnología". ¿En qué piensas } \\
\text { cuando escuchas esa palabra? }\end{array}$} \\
\hline $\begin{array}{l}\text { Representaciones generales } \\
\text { relación mundo social/ } \\
\text { tecnologías }\end{array}$ & \multicolumn{2}{|c|}{$\begin{array}{l}\text { ¿Ha cambiado el mundo con las tecnologías actuales? } \\
\text { ¿De qué manera? }\end{array}$} \\
\hline $\begin{array}{l}\text { Atribución éxito/fracaso social } \\
\text { de tecnologías y sus causas }\end{array}$ & \multicolumn{2}{|c|}{$\begin{array}{l}\text { A tu juicio, cuáles son las tecnologías más populares, } \\
\text { las que más se usan en tu medio. ¿Por qué crees que } \\
\text { ello es así? }\end{array}$} \\
\hline $\begin{array}{l}\text { Relación general tecnologías/ } \\
\text { individuo/vida cotidiana }\end{array}$ & \multicolumn{2}{|c|}{$\begin{array}{l}\text { ¿Qué tecnologías utilizas en tu vida cotidiana y qué } \\
\text { haces con ellas? }\end{array}$} \\
\hline $\begin{array}{l}\text { Representaciones generales } \\
\text { relación individuo/tecnologías }\end{array}$ & \multicolumn{2}{|c|}{$\begin{array}{l}\text { ¿Ha cambiado tu vida con las tecnologías que utilizas } \\
\text { día a día? ¿De qué manera? }\end{array}$} \\
\hline $\begin{array}{l}\text { Atribución éxito/fracaso } \\
\text { individual de tecnologías y sus } \\
\text { causas }\end{array}$ & \multicolumn{2}{|c|}{$\begin{array}{l}\text { ¿Qué tecnologías te resultan más y menos atractivas? } \\
\text { ¿De qué depende tu elección? }\end{array}$} \\
\hline $\begin{array}{l}\text { Negociación de tiempos y } \\
\text { rutinas de ocio }\end{array}$ & \multicolumn{2}{|c|}{$\begin{array}{l}\text { ¿Qué haces en tus tiempos de ocio? ¿Involucras algún } \\
\text { tipo de tecnología o plataforma digital? } \\
\text { ¿Percibes alguna influencia de tus profesores, amigos } \\
\text { y compañeros? }\end{array}$} \\
\hline $\begin{array}{l}\text { Negociación de procesos de } \\
\text { aprendizaje y de producción de } \\
\text { conocimiento }\end{array}$ & \multicolumn{2}{|c|}{$\begin{array}{l}\text { ¿Cómo organizas tus rutinas y tiempos de estudio? } \\
\text { ¿Involucras algún tipo de tecnología o plataforma digital? } \\
\text { ¿Percibes alguna influencia de tus profesores, amigos } \\
\text { y compañeros? }\end{array}$} \\
\hline $\begin{array}{l}\text { Amalgama de significados } \\
\text { de las estructuras culturales } \\
\text { en que se desenvuelven } \\
\text { estudiantes y docentes }\end{array}$ & \multicolumn{2}{|c|}{$\begin{array}{l}\text { ¿Qué diferencias y similitudes encuentras entre lo que } \\
\text { dicen tus profesores con respecto a las TIC e Internet } \\
\text { y lo que dicen tus compañeros? ¿Con cuál grupo te } \\
\text { identificas más? }\end{array}$} \\
\hline $\begin{array}{l}\text { Relación e intercambio entre } \\
\text { actores y audiencias }\end{array}$ & \multicolumn{2}{|c|}{$\begin{array}{l}\text { ¿Consideras que las ideas que tienen tus maestros so- } \\
\text { bre las tecnologías son válidas e influyentes? Justifica } \\
\text { tu respuesta }\end{array}$} \\
\hline $\begin{array}{l}\text { Subculturas, patrones y objetos } \\
\text { de identificación }\end{array}$ & \multicolumn{2}{|c|}{$\begin{array}{l}\text { Describe las prácticas más comunes, mediadas por TIC } \\
\text { e Internet, de los estudiantes en el aula de clase. ¿Qué } \\
\text { sentido tienen estas? }\end{array}$} \\
\hline $\begin{array}{l}\text { Percepción de orden } \\
\text { cooperativo versus orden } \\
\text { coercitivo }\end{array}$ & \multicolumn{2}{|c|}{$\begin{array}{l}\text { ¿Qué tecnologías utilizas por obligación y cuáles por } \\
\text { gusto? } \\
\text { ¿De qué o quiénes depende esa diferencia? }\end{array}$} \\
\hline
\end{tabular}

Fuente: elaboración propia 
En este sentido, cuando preguntamos por lo que cada uno de ellos pensaba de las tecnologías en general y las TIC en particular, abundaron las definiciones generales, asociadas a la facilitación de tareas y en un tono optimista. Nos encontramos, entonces, con comentarios como estos:

- La tecnología es un conjunto de herramientas que se están renovando activamente y que ayudan al ser humano a mejorar sus actividades diarias (...), esto obliga a que las cambiemos porque van a ser dejadas de lado (renovadas) (G.F.1., 2013).

- Lo primero que a mí se me viene a la mente son los avances, la facilidad a la hora de hacer las cosas, las grandes posibilidades que podemos llegar a hacer con eso (Ídem).

- Se me viene a la mente la palabra "innovación"; es como algo de avance, es como algo que va a mejorar (Ídem).

- (La tecnología) facilita las actividades cotidianas, que está en constante evolución, que cambia y que siempre va mejorando; y comunicación (Ídem).

Los sujetos reconocieron, sin embargo, la existencia de ciertas influencias discursivas en lo que atañe a su relación con las TIC por parte de cuatro grupos predominantes: la publicidad, la familia, la Universidad y los compañeros. No obstante, afirmaron también que la validez y facticidad otorgada a cada uno de esos discursos variaba según las necesidades o demandas de la situación concreta en la que se encontrasen. En palabras de algunos estudiantes:

...hay mucho consumismo, creo que estamos bombardeados de mucha publicidad en los medios de transporte, en los celulares, más que todo donde hay Internet, televisión, entonces nos volvemos consumistas, digamos si vemos algo más avanzado dejamos de lado lo que utilizamos y pues eso es ser como ser civilizado ahorita, entonces pues ya estamos creando, o sea, no hay una consciencia de sino de desechar por desechar, no hay una consciencia de la utilización que verdaderamente se le debe dar a un aparato, también que las personas tienen menos tiempo de ocio entonces el descanso ya no es como las horas que tienen que dormir tanto tiempo sino que me la paso en face tanto tiempo, después hago trabajos, etc. (G.F.3., 2013).

En mi casa son muy tecnológicos y en ocasiones por ejemplo llega la hora de la comida, mi mamá por el teléfono me escribe "a comer" entonces uno dice como: ¿qué? -risas- ¡si estamos en la misma casa! (G.F.1., 2013).

La Influencia de amigos, la sociedad o el ambiente laboral para que uno tenga un celular para estar en contacto genera la necesidad de que el otro también lo tenga (G.F.2., 2013b). 
Las tecnologías de punta te dan un estatus (...), además, el tema social te crea la necesidad de estar conectado con la gente. Necesitas ser reconocido. A veces las tecnologías de la información y el Facebook te alimentan el ego (Ídem).

Soy un usuario frecuente de Internet, lo uso todos los días que estoy en casa. También tiene estrecha relación con la Universidad (...), con los compañeros de la Universidad (para) discutir algunas cosas en cuestiones del panorama político actual de los que se discuten en las redes sociales, es algo que me consume algún tiempo: los amigos, la familia, sobre todo los que están lejos -fuera del país-, hablo mucho con ellos (Ídem).

Lo que decía Diana: todo el mundo con Iphone y con Black Berry y uno con celular ahí todo feíto, da pena sacarlo o que lo llamen a uno, ¡huy no! (G.F.6., 2013).

Asimismo, los sujetos se expresaron en términos conflictivos y dicotómicos con respecto a lo que para ellos representaban las TIC. Además de asociar las TIC a innovación, eficiencia, ubicuidad y portabilidad, las asociaron también a consumismo, moda, facilismo, aislamiento y dependencia, otorgándole mucha importancia a la autonomía personal:

Yo creo que el mundo ha cambiado drásticamente con el uso de las tecnologías, o sea uno ve cada día que es muchísimo más el cambio que se da más que todo en la parte social, porque creo que se ha generado una ruptura muy seria frente a las relaciones interpersonales, (en tanto) que las personas que tienen un uso masivo de las tecnologías prácticamente están aisladas de las comunidades con las que comparten. Yo no me siento así porque mantengo muchas relaciones interpersonales con muchas comunidades, amigos. Pero hay otras personas que uno si las ve (dependientes). Por ejemplo, una pareja cenando y (al mismo tiempo chateando) están tan cerca pero a la vez tan distanciados (...), siento que eso ha afectado mucho la parte social (...), la gente se siente feliz que las tecnologías estén aplicadas en el mundo (...), se siente contento que con el Internet... (G.F.1., 2013).

...(La tecnología) es mejor para buscar información, pero también tienen aspectos negativos (...) separó mucho a las familias, cada uno es más individualista, cada uno está con su aparato electrónico. (Las familias) ya no se reúnen como antes; también (las tecnologías) clasificaron mucho la sociedad, el prestigio (de) las personas (se determina) por los aparatos que tienen. (También) es una herramienta importante para los sectores privados, la policía, la DIAN (pues) les permite desarrollar sus actividades fácilmente. (Por ejemplo) o sea la policía está buscando a alguien que se desapareció y tenía en el carro un GPS pues le queda más fácil ubicarlo que antes que no tenía nada de eso (G.F.2., 2013b). 
En cierta parte (las TIC) facilitan algunas cosas, pero ya depende del uso que uno le quiera dar porque nos hemos vuelto muy conformistas. Entonces ya está el Internet y la gente de ahí saca todo. Que ir más allá, como esforzarse por hacer las cosas porque entonces uno se vuelve facilista (Ídem).

Yo detecto como un conflicto. Tengo una contradicción porque una de las tecnologías que más me resulta atractiva es el celular, precisamente por lo que hemos hablado, pero a la vez se me hace peligrosa porque las personas, las que no saber portar un celular, dependen de ellas (Ídem).

...(Internet) es bueno y es malo si uno sabe manejarlo. En Facebook uno está hablando con una persona pero si al lado hay otra uno no le pone atención a ella porque está hablando por el Facebook (...), en mi familia siempre son así, se la pasan conectadas a esos aparatos y uno les habla y nada (G.F.5, 2013).

De hecho, los sujetos fueron mucho más detallados en la descripción de lo que a su juicio eran los peligros que entrañaban las TIC, generándose tras cada intervención un consenso que se manifestaba en gestos de asentimiento, risas, y comentarios a propósito de lo que se estaba diciendo. En este punto, abundaron las referencias a la moda, la globalización y el capitalismo como referentes de consumismo, y el debilitamiento de la sociabilidad presencial por cuenta de una constante comunicación online:

...la tecnología nos la venden como el hecho de tener algo mejor que lo que tiene el otro (G.F.6., 2013).

La cosa que uno tiene un computador de tal manera con tal programa y luego sale otro programa y uno lo quiere para estar a la altura de la tecnología de otra persona (F.G.7., 2013).

Esta época lo que es importante por así decirlo es la cibernética, lo que nos mantiene conectados, la máquina. Es el boom del momento, la producción en serie (G.F.1., 2013).

Lo que me pasa a mí en Internet es ponerme a ver videos estúpidos -risas-, es algo estúpido no sé por qué uno gasta tanto tiempo en eso. Es tiempo de ocio bobo o en televisión uno ve puras bobadas y pierde tiempo y pierde todo un fin de semana donde uno puede hacer muchas cosas, y después deja todo por eso (por estar conectado). No sabemos utilizar (las tecnologías) aquí en Colombia (...): llegamos tarde a todo, no hacemos las cosas y cuando nos damos cuenta se ha pasado el tiempo y lo hacemos rápido y mediocre (Ídem).

Estamos en un país capitalista, la globalización es capitalista (...), la globalización hace que se acerquen más entre países, entre 
ciudades. Así empezó este cuento del orden en Estados Unidos, se facilita más el intercambio de bienes y servicios. (Esto) hace que lleguen acá (a Colombia) los productos (...). Tenemos un contacto más central con Estados Unidos y otros países (...), todos estamos en el mismo punto, todos tenemos las mismas tecnologías (que nos permiten) avanzar en este mundo globalizado (G.F.3., 2013b).

Nosotros queremos adoptar unas culturas que no son propias de nosotros. También influye lo de la globalización (...); (o como producto de la moda). Por ejemplo, por que digamos hace más o menos un año y medio todo el mundo tenia BlackBerry y ahora todo el mundo tiene Iphone y todo el mundo empieza a cambiar a Iphone porque tiene mejores especificaciones. Porque BlackBerry cayó y se vuelve más de moda que por necesidad (G.F.3., 2013).

Algo similar sucedió con los tiempos de ocio, en donde los sujetos diferenciaron actividades online y offline. Al respecto, manifestaron tener mayor estima por las actividades presenciales y al aire libre, aun cuando estas se organizaban vía redes sociales o aplicaciones móviles. Asimismo, reconocieron pasar la mayor parte de su tiempo de ocio conectados a Internet, desde donde accedían tanto a redes sociales como a servicios de radio y televisión. En este punto, le asignaron una influencia decisiva a amigos y compañeros en el diseño de esas prácticas, como se subraya en las siguientes opiniones:

(La tecnología) es importante, es buena porque la tecnología es para todo, pero no es tan bueno porque uno se involucra demasiado en el tema (la tecnología) y deja de hacer otras cosas (como) hacer (actividades) al aire libre (...). Hay muchas redes sociales en las que uno tiene amigos, pero no es como tener el contacto personal (G.F.1., 2013).

Recuerdo que una vez fui a un restaurante (y observé a dos personas) Los dos estaban así tecleando, ni se miraban (...). Uno sale para un momento de ocio, para entretenerse, hablar: ¡no hay cosa más chévere que hablar con una persona! Yo a veces prefiero gastar un minuto (de celular) y hablar con una persona (...). Me parece que es importante el celular pero hasta cierto punto, hay que saber dividir en qué momento (es un contacto) personal (y cuando se convierte en) impersonal (Ídem).

Uno puede salir a jugar, digamos yo personalmente todos los sábados salgo a jugar fútbol pero salgo a jugar sino estoy conectado, igual, me conecto y me da pereza salir porque quiero quedarme conectado (G.F.4., 2013).

Bueno pues yo en ese sentido lo hago como muy combinado, procuro digamos a veces estar en el parque: con el perro, con mi familia. Pero igual la mayoría del tiempo que estoy en mi casa: viendo televisión, jugando video juegos, escuchando música o 
chateando con mis amigos. Es que la tecnología es un punto súper importante (...). De hecho todo el día uno ya está usando (celular) la tecnología viviendo a través de esos medios (Ídem).

Los espacios académicos se presentaron, además, como diferenciados de los tiempos de ocio. Los sujetos describieron a sus profesores como figuras de autoridad científica, agentes que trazan límites y restricciones en los usos de las TIC en el aula, y generadores de protocolos para la investigación mediada por las TIC, como se puede detallar en los siguientes sentires:

Los profesores pienso que obviamente en una institución, dicen que uno no puede estar chateando en el celular, lo dicen porque uno se desconcentra de la clase. Hay restricciones, en la Universidad, en las salas de cómputo, no se puede acceder a YouTube o a ciertas páginas como Facebook (G.F.8., 2013).

Los docentes han tenido influencia porque al ellos manejar este tipo de medios lo incitan a uno a usarlos, entonces toca aprender a manejarlos y los amigos también influyen. Pero en mi caso ha sido más empírico (G.F.7., 2013).

Los profesores porque son los que dan las fechas de entrega, ellos son los que dan las pautas durante la semana, es la influencia del profesor la que indica los tiempos de estudio (Ídem).

En principio, los sujetos generaron una relación directa entre las oposiciones ocio/academia, gusto/obligación, y redes sociales/Moodle.

Por obligación el Moodle, porque es un requisito de la Universidad para trabajos, notas, avisos, eventos de la Universidad. Por gusto Facebook y YouTube, Monopolio Urbano, una página donde me llegan las canciones de los estrenos y yo las escucho antes de que salgan (G.F.4., 2013).

...es claro saber para qué se usan las redes sociales y para qué se usa el Moodle (...). Moodle es para uso académico, creo yo, y las redes sociales, aunque a veces ayudan a comunicarnos para lo académico, están más enfocadas en lo social (hablar, saber de la vida de los demás, chismosear), entonces para mí es clara la diferencia (Ídem).

Sin embargo, dicha relación entró en conflicto cuando se les preguntó específicamente si su presencia en redes sociales en tiempos de ocio obedecía más a un principio de gusto que de necesidad. Así se expresaron algunos de los sujetos:

Ahorita a muchos de nosotros en la utilidad uno cuenta todo: el Twitter, el Facebook sirve para narrar todo, es por uno. Soy adicto a las redes sociales y eso, a que voy a hacer tal cosa y no es porque nadie más lo obligue sino es por contarle al mundo lo que uno va a hacer, ¡es algo loco! (G.F.1., 2013). 
Pues se han dado como las dos posturas: (primera) algunos profesores ni siquiera el Moodle les gusta, dicen que uno debería estudiar como en sus tiempos, porque aprendían más con los libros, leyendo, asistiendo a bibliotecas, sin tener que buscar todo por Internet, (segunda) como hay muchos que se apoyan con las tecnologías en clase y esas cosas y se enfocan más en saberlas manejar y no estar con los distractores: las aplicaciones, las redes sociales o lo que no competa a la clase, y creo que en algunos casos tanto profesores como compañeros mantienen las mismas prácticas. Es la misma cosa como consecuencia de los adelantos de la tecnología que lo llevan estar a uno ahí. (Por esta razón) las (personas) muy poco van a las bibliotecas. A esa tendencia están sometidos tanto profesores como alumnos (G.F.2., 2013).

No encuentro cuáles son las que uno tiene que usar por obligación; todo es ya como por gusto. ¡Ah no! ¡Pero! Moodle ¡No!, ¡no! (Ídem).

Finalmente, manifestaron su incomodidad y desconcierto ante las políticas de restricción de sitios web (Facebook, YouTube, Twitter) que ha establecido la Universidad, y que estos interpretaron como desconocimiento por parte de la institución de los canales a través de los cuales se accede hoy en día a la información.

(Las redes sociales) contribuyen a los procesos formativos porque son una herramienta bastante útil, por el acceso que tenemos al acceso de información global e instantánea. Pero tiene limitaciones en cuanto a que empresas y colegios tienen una serie de páginas bloqueadas porque decimos que lo académico se convierte en ocio, y como dicen otros que el YouTube es de ocio y pueden usarlo para otras cosas, entonces asumen que acceden solo para lo del ocio, y docentes han solicitado que desbloqueen el Facebook porque ellos lo están utilizando académicamente como comunidad (...). Entonces, ¿por qué solo verlo como ocioso? Si se le puede sacar un provecho académico y eso lo he escuchado más a los profesores (...), porque es una plataforma de comunicación para otro tipo de cosas: ofertas de empleo, reuniones de interés de la facultad (G.F.2., 2013b).

En términos de los problemas que tienen es que básicamente el sistema escolar está separado de la tecnología, el sistema de calificación, los modelos pedagógicos, y no tiene una conexión con los modelos tecnológicos, esto del trabajo de la realidad ampliada para contenidos de biología y química, y hay profesores del siglo XVIII para estudiantes del siglo XXI. Entonces no hay una relación directa en eso, y oportunidad es un espacio para crear conocimiento y difundir proyectos que uno tenga, apuestas a nivel personal (Ídem).

Creo que las redes sociales son muy importantes para el aprendizaje (...). Facebook es una quimera, creo que uno puede adquirir conocimiento y compartirlo, podemos referenciar muchas cosas 
en donde nos podemos basar. Por ejemplo, otra red social que es como una adicción para mí es YouTube, porque es una red social en donde aprendo, así fue como pasé mis cursos de cálculo, me empiezan a mostrar cómo se hace el ejercicio, pauso el video y sigo haciendo el ejercicio, he aprendido cómo instalar juegos, programas, despinchar una llanta, he aprendido cómo hacer ejercicio (...), puedo ver opiniones de personas de España, cosas en otro idioma con subtítulos de los noticieros, veo documentales para estudiar geología: el profesor nos colocaba el link de YouTube y empezaba a explicar lo de la tierra (Ídem).

\section{Conclusiones}

Según los resultados antes descritos, podría pensarse que la educación emerge como espacio en el que forzosamente convergen: (a) narrativas sobre nuestro tiempo y sobre la centralidad del fenómeno tecnológico en el cambio de época que vivimos, y (b) de distintos actores con arraigos generacionales y disciplinares igualmente diversos. La Universidad resultaría así ser un laboratorio cultural que se presta tanto para la creatividad como para la regulación normativa (Bauman, 2002). De ahí que las prácticas educativas puedan entenderse como una serie de actos y representaciones (performance) compuestas de narrativas básicas, intérpretes, medios de producción simbólica y puestas en escena legitimadas o en conflicto con la sociedad en la que acontecen. En este sentido, se identificaron tres dinámicas que permitirían comprender los vínculos entre mediaciones culturales y prácticas mediáticas: (1) el establecimiento de códigos y protocolos, (2) la búsqueda de re-fusión, es decir, la intención de los actores de hacer exitosas sus interpretaciones sobre el significado de fenómenos considerados por ellos relevantes, y (3) procesos de negociación entre estudiantes en lo relativo a los usos de las tecnologías, muchos de los cuales escapan a la mirada del docente.

El uso de enunciaciones dicotómicas es constante, lo que lleva a pensar que la tecnología está enmarcada por consideraciones binarias recurrentes en las intervenciones de los estudiantes. En lo que se refiere a las TIC, las posturas de los sujetos denotaban la convivencia de convenciones apocalípticas e integradas (Eco, 1965) de carácter tecnológico y sociocultural. Su uso se hizo extensivo al análisis de los tiempos de ocio y aprendizaje, situación que podrían estar reforzando tanto los docentes como la institución al privilegiar (o restringir) el uso de determinadas plataformas o prácticas académicas, informáticas y comunicativas. Podría ser que la interpretación del fenómeno tecnológico en clave binaria sea una forma de reflexividad transversal a los niveles agencial, institucional-educativo, científico y mediático, en tanto es una forma elemental (Durkheim, 1993) de la vida cultural.

De acuerdo a los resultados arriba expuestos, son básicamente cinco los agentes con capacidad de configuración de los procesos educativos: los medios (en especial la publicidad), la familia, la institución universitaria, los docentes y los 
mismos estudiantes. Enfatizamos en que la validez que los sujetos otorgaron a los medios, la familia, la Universidad y los docentes variaba en función de las circunstancias concretas en las que se encontrasen. No tanto así en lo que respecta a la relación de los sujetos con sus pares, cuya influencia es evidente y constante, más allá de tiempos (ocio o academia), escenarios (redes sociales, Moodle o aula de clases) y actividades concretas.

Cabe señalar que hubo dos dispositivos que destacaron como catalizadores de la discusión: los computadores portátiles y los teléfonos móviles. Los primeros por sus prestaciones, eficiencia y carácter multimedial, y los segundos debido a su portabilidad, su aura innovadora y como símbolo de estatus. Asimismo, las redes sociales fueron descritas como espacios que permiten la habitabilidad de Internet en tanto se comparte información, se construye una identidad y se generan, mantienen o transforman las relaciones sociales.

Una de las claves en términos de educación mediática y competencia digital podría ser, como lo sugiere uno de los comentarios de los estudiantes, definir el tipo de tecnologías que se deben incorporar en función de las prácticas que se buscan promover, los sujetos de esas prácticas, y los entornos y necesidades institucionales. En este sentido, la inversión en TIC debe ir acompañada de criterios claros sobre la cultura material que se desea consolidar en cada institución.

\section{Referencias}

Alexander, J. (2000). La promesa de una sociología cultural. Discurso tecnológico y la máquina de la información sagrada y profana. En J. Alexander, Sociología cultural: formas de clasificación en las sociedades complejas, 165-201. España: Anthropos-Flacso.

. (2000b). Las teorías sociológicas desde la segunda guerra mundial. Barcelona: Gedisa.

. (2003). The Meanings of Social Life: A Cultural Sociology. New York: Oxford University Press.

. (2005). Performance and Power. Newsletter of the Sociology of Culture, 20(1), 1-5.

. (2005b). Pragmática cultural: un nuevo modelo de performance social. Revista Colombiana de sociología, 24, 9-67.

. (2008). Clifford Geertz and the Strong Program: The Human Sciences and cultural sociology. Cultural Sociology, 2, 157-168.

. (2008b). Iconic consciousness: the material feeling of meaning. Environment and Planning D. Society and Space, 26, 728-794. 
(2008c). Iconic Experience in Art and Life: Surface/Depth Beginning with Giacometti's Standing Woman. Theory, Culture and Society, 25(1).

(2009). What We Learn from the Humanities? Culture, 23(1), 4-8.

Balsamo, A. (2011). Designing Culture. The Technological Imagination at Work. Duke University Press.

Barrera-Osorio, F., y Linden, L. (2009). The use and misuse of computers in education: evidence from a randomized experiment in Colombia. In Policy Research Working Paper. 4836. Impact Evaluation, Series No. 29. The World Bank, Human Development Network.

Baudrillard, J. (2012). El sistema de los objetos. México: Siglo XXI editores.

Bauman, Z. (2002). La cultura como praxis. Buenos Aires: Paidós.

Carr, N. (2011). ¿Qué está haciendo Internet con nuestras mentes? Superficiales. Bogotá: Taurus.

Cobo, C. (2009). Strategies to Promote the Development of E-competencies in the Next Generation of Professionals: European and International Trend. México: Skope-Flacso.

Cobo, C., y Kuklinski, H. P. (2007). Planeta Web 2.0. Inteligencia colectiva o medios fast food. Barcelona/México D.F: Grup de Recerca d'Interaccions Digitals, Universitat de Vic.Flacso.

Castells, M. (2006). La Sociedad Red. Madrid: Alianza Editorial.

Durkheim, E. (1993). Las formas elementales de la vida religiosa. Madrid: Alianza.

Eco, U. (1965). Apocalípticos e Integrados. Barcelona: Lumen.

G.F.1. (20 de abril de 2013). Nivel 1: Generalidades. Tecnología, Sociedad e Individuo. (J. S. López, D. S. Franco, entrevistadores, y J. D. López, editor). Bogotá.

G.F.2. (28 de abril de 2013). Nivel 1: Generalidades. Tecnología, Sociedad e Individuo. (J. S. López, D. S. Franco, entrevistadores, y J. D. López, editor). Bogotá.

G.F.3. (6 de mayo de 2013). Nivel 1: Generalidades. Tecnología, Sociedad e Individuo. (J. S. López, D. S. Franco, entrevistadores, y J. D. Gómez, editor). Bogotá.

G.F.4. (14 de mayo de 2013). Nivel 1: Generalidades. Tecnología, Sociedad e Individuo. (J. S. López, D. S. Franco, entrevistadores, y J. D. Gómez, editor). Bogotá.

G.F.5. (15 de septiembre de 2013). Nivel 2: Negociación entre actores. (J. S. López, D. S. Franco, entrevistadores, y J. D. Gómez, editor). Bogotá.

G.F.6. (24 de septiembre de 2013). Nivel 2: Negociación entre actores. (J. S. López, D. S. Franco, entrevistadores, y J. D. Gómez, editor). Bogotá. 
G.F.7. (1 de octubre de 2013). Nivel 2: Negociación entre actores. (J. S. López, D. S. Franco, entrevistadores, y J. D. Gómez, editor). Bogotá.

G.F.8. (20 de octubre de 2013). Nivel 2: Negociación entre actores. (J. S. López, D. S. Franco, entrevistadores, y J. D. Gómez, editor). Bogotá.

Elster, J. (2010). La explicación del comportamiento social. Más tuercas y tornillos para las ciencias sociales. Barcelona: Gedisa.

Ferrés, J., y Piscitelli, A. (2011). La competencia mediática: propuesta articulada de dimensiones e indicadores. Comunicar, 38(19), 75-82.

Ito, M., Matteo, B., Danah, B., Becky, H.-S., Patricia, L., Laura, P. C., y Horst, H. (2009). Living and Learning with New Media: Summary of Findings from the Digital Youth Project. (T. J. Learning, ed.). Cambridge: MIT Press.

Jenkins, H. (2008). Convergence Culture. La cultura de la convergencia de los medios de comunicación. Barcelona: Paidós.

. (2009). Fans, blogueros y videojuegos. La cultura de la colaboración. Barcelona, Paidós.

Kangas, S. (2011). Digital Pioneers. Tracing the cultural drivers of future media culture. Helsinki: Nuorisotutkimusverkost.

López, J. S., y Santiago, D. (2012). Filosofía para Niños y Competencias Digitales. Aportes para la Educación Formal en la era de información. En V. C. Educação (ed.). Rio de Janeiro: UERJ.

Melucci, A. (2001). Vivencia y convivencia. Teoría social para una era de la información. Madrid: Trotta.

Moratalla, T. D. (2007). Bioética y hermenéutica. La aportación de Paul Ricoeur a la bioética. Veritas, 2(17), 281-312. Recuperado el 20 de septiembre de 2013 de http://www.fondsricoeur.fr/photo/bioetica.pdf

Reed, I., y Alexander, J. (2009). Social Science as Reading and Performance. A Cultural-Sociological Understanding of Epistemology. European Journal of Social Theory, 12(1), 21-41.

Pisticelli, A., Adaime, I., y Binder, I. (2010). El proyecto Facebook y la Posuniversidad. Sistemas operativos sociales y entornos abiertos de aprendizaje. Buenos Aires: Ariel.

Ricoeur, P. (2006). La Vida: un relato en busca de narrador. Ágora, 25(2), 9-22. . (2000). Narratividad, fenomenología y hermenéutica. Anàlisi, (25), 189-207.

. (2005). Volverse capaz, ser reconocido. (s.d. Revue des revues de l'adpf, ed.). Recuperado el 15 de julio de 2012, de Ministerio de Asuntos Exteriores 
de Francia http://www.diplomatie.gouv.fr/fr/IMG/pdf/Revue_des_ revues_200_112B78.pdf

Scolari, C. (2010). Ecología de los medios. Mapa de un nicho teórico. Quaderns del CAC, 34(13), 17-25.

Strauss, A. y Corbin, J. (2002). Bases de la investigación cualitativa. Técnicas y procesos para desarrollar la teoría fundamentada. Medellín: Universidad de Antioquia.

Unesco. (2005). Hacia las sociedades del conocimiento. Jouve: Unesco.

Wolton, D. (2000). Internet, ¿y después? Una teoría crítica de los nuevos medios de comunicación. Barcelona: Gedisa.

Zabludovsky, G. (1995). Clásicos y contemporáneos de la teoría sociológica. Entrevista con Jeffrey Alexander. En Sociología y política, el debate clásico y contemporáneo. México: Editorial Miguel Ángel Porrúa. 